\title{
A Note on Edge-Domsaturation Number of a Graph
}

\author{
Devadhas Nidha, Murugan Kala \\ Department of Mathematics, Manonmaniam Sundaranar University, Tirunelveli, India \\ Email: nidhamaths@gmail.com, karthipyi91@yahoo.co.in
}

Received April 27, 2012; revised May 30, 2012; accepted June 12, 2012

\begin{abstract}
The edge-domsaturation number $d s^{\prime}(G)$ of a graph $G=(V, E)$ is the least positive integer $k$ such that every edge of $G$ lies in an edge dominating set of cardinality $k$. In this paper, we characterize unicyclic graphs $G$ with $d s^{\prime}(G)=q-\Delta^{\prime}(G)$ +1 and investigate well-edge dominated graphs. We further define $\gamma^{\prime}$-critical, $\gamma^{\prime+}$-critical, $d s^{\prime}$-critical, $d s^{\prime+}$-critical edges and study some of their properties.
\end{abstract}

Keywords: Edge-Dominating Set; Edge-Domination Number; $d s^{\prime}$-Critical; Edge-Domsaturation Number; Well Edge Dominated Graph

\section{Introduction}

Throughout this paper, $G$ denotes a graph with order $p$ and size $q$. By a graph we mean a finite undirected graph without loops or multiple edges. For graph theoretic terms we refer Harary [1] and in particular, for terminology related to domination theory we refer Haynes et al. [2].

\subsection{Definition}

Let $G=(V, E)$ be a graph. A subset $D$ of $E$ is said to be an edge dominating set if every edge in $E-D$ is adjacent to at least one edge in $D$. An edge dominating set $D$ is said to be a minimal edge dominating set if no proper subset of $D$ is an edge dominating set of $G$. The edge domination number $\gamma^{\prime}(G)$ of a graph $G$ equals the minimum cardinality of an edge dominating set of $G$. An edge dominating set of $G$ with cardinality $\gamma^{\prime}(G)$ is called a $\gamma^{\prime}(G)$-set or $\gamma^{\prime}$-set.

Acharya [3] introduced the concept of domsaturation number $d s(G)$ of a graph. For any graph $G$ of order $p$, and for any integer $r$ such that $\gamma(G) \leq r \leq p$, we call the set $D C_{r}(G)=\left\{u \in V(G) / u \in D\right.$ forsome $\left.D \in \mathfrak{A}_{r}(G)\right\}$ the $r$-level domination core of $G$. We say that $G$ is $r$-level domination-saturated (or in short, " $r$-domsaturated") if $D C_{r}(G)-V(G)$. The domsaturation number $d s(G)$ is then defined by $d s(G)=\min \{r / G$ isr-domsaturated $\}$. Arumugam and Kala [4] observed that for any graph $G$,

$d s(G)=\gamma(G)$ or $d s(G)=\gamma(G)+1$ and obtained several results on $d s(G)$. We now extend the concept of domsaturation number of a graph to edges.

\subsection{Definition}

The least positive integer $k$ such that every edge of $G$ lies in an edge dominating set of cardinality $k$ is called the edge-domsaturation number of $G$ and is denoted by $d s^{\prime}(G)$.

If $G$ is a graph with edge set $E$ and $D$ is a $\gamma^{\prime}$-set of $G$, then for any edge $e \in E-D, D \cup\{e\}$ is also an edge dominating set and hence $d s^{\prime}(G)=\gamma^{\prime}(G)$ or $\gamma^{\prime}(G)+1$.

Thus we have the following definition.

\subsection{Definition}

A graph $G$ is said to be of class 1 or class 2 according as $d s^{\prime}(G)=\gamma^{\prime}(G)$ or $\gamma^{\prime}(G)+1$.

\subsection{Definition}

An edge $e$ of $G$ is

1) $\gamma^{\prime}$-critical if $\gamma^{\prime}(G-e) \neq \gamma^{\prime}(G)$;

2) $\gamma^{\prime+}$-critical if $\gamma^{\prime}(G-e)>\gamma^{\prime}(G)$;

3) $\gamma^{\prime}$-critical if $\gamma^{\prime}(G-e)<\gamma^{\prime}(G)$;

4) $\gamma^{\prime}$-fixed if every $\gamma^{\prime}$-set contains $e$;

5) $\gamma^{\prime}$-free if there exists $\gamma^{\prime}$-sets containing $e$ and also $\gamma^{\prime}$-sets not containing $e$;

6) $\gamma^{\prime}$-totally free if there is no $\gamma^{\prime}$-set containing $e$.

We use the following theorem.

\subsection{Theorem [5]}

For any connected unicyclic graph $G=(V, E)$ with cycle $C, \gamma^{\prime}(G)=q-\Delta^{\prime}(G)$ if and only if one of the following holds.

1) $\mathrm{C}=\mathrm{C}_{3}$;

2) $G=C_{3}=\left(u_{1}, u_{2} . u_{3}, u_{1}\right), \operatorname{deg} u_{1} \geq 3, \operatorname{deg} u_{2}=\operatorname{deg} u_{3}=2$, $\operatorname{deg}\left(u_{1}, w\right) \leq 2$ for all vertices $w$ not on $C$ and $\operatorname{deg} w \geq 3$ for at most one vertex $w$ not on $C$;

3) $G=C_{3}=\left(u_{1}, u_{2} . u_{3}, u_{1}\right), \operatorname{deg} u_{1} \leq 3, \operatorname{deg} u_{2} \leq 3, \operatorname{deg} u_{3}=2$ 
all the vertices not on $C$ adjacent to $\mathrm{u}_{1}$ have degree at most 2 and all vertices whose distance from $u_{1}$ is 2 are pendent vertices;

4) $C=C_{3}=\left(u_{1}, u_{2}, u_{3}, u_{1}\right), \operatorname{deg} u_{1}=3, \operatorname{deg} u_{2} \leq 3, \operatorname{deg} u_{3} \leq 3$ and all vertices not on $C$ are pendent vertices;

5) $C=C_{4}$;

6) $C=C_{4}$, either exactly one vertex of $C$ has degree at least 3 and all vertices not on $C$ are pendent vertices.

\section{Main Results}

\subsection{Lemma}

An edge $e$ of $G$ is $\gamma^{\prime}$-critical if and only if $\gamma^{\prime}(G-e)=\gamma^{\prime}(G)-1$

\section{Proof}

For any edge $e$, we observe that $\gamma^{\prime}(G-e)=\gamma^{\prime}(G)-1$ or $\gamma^{\prime}(G)$ or $\gamma^{\prime}(G)+1$. Now, suppose $e$ is $\gamma^{\prime}$-critical. Then $\gamma^{\prime}(G-e)<\gamma^{\prime}(G)$. Hence $\gamma^{\prime}(G-e)=\gamma^{\prime}(G)-1$. The converse is obvious.

\subsection{Theorem}

An edge $e$ is $\gamma^{\prime}$-critical if and only if

$$
N(e) \subset \cup_{f \in D-e} N(f)
$$

for some $\gamma^{\prime}$-set $D$ containing $e$.

\section{Proof}

If $e$ is $\gamma^{-}$-critical, $\gamma^{\prime}(G-e)=\gamma^{\prime}(G)-1$ by lemma 2.1. Let $S$ be a $\gamma^{\prime}$-set of $G-e$. If $S$ contains an edge of $N(e)$, then $S$ will be an edge dominating set of $G$ and hence $\gamma^{\prime}(G) \leq \gamma^{\prime}(G-e)$, a contradiction. Thus $S$ does not contain any edge of $N(e)$. Since $\gamma^{\prime}(G-e)=\gamma^{\prime}(G)-1$, $D=S \cup\{e\}$ is a $\gamma^{\prime}$-set of $G$ and so Equation (1) holds. Conversely, suppose $e$ is an edge such that (1) is true. Then $G-e$ is an edge dominating set of $G-e$ and hence $\gamma^{\prime}(G-e)=\gamma^{\prime}(G)-1$. Thus $e$ is $\gamma^{\prime-}$-critical.

\subsection{Theorem}

Let $G$ be a graph without isolated edges. An edge $e$ in $G$ is $\gamma^{\prime-}$-critical if and only if

1) $e$ is $\gamma^{\prime}$-free, and

2) no $\gamma^{\prime}$-set of $G-e$ contains any edge of $N(e)$.

\section{Proof}

If $e$ is $\gamma^{\prime-}$-critical, then $\gamma^{\prime}(G-e)=\gamma^{\prime}(G)-1$ by Lemma 2.1. As in theorem 2.2, if $S$ is any $\gamma^{\prime}$-set of $G-e$, then $S$ will not contain any edge of $N(e)$ and $S \cup\{f\}$ is a $\gamma^{\prime}$ set of $G$ for every $f \in N[e]$. This implies that $e$ is $\gamma^{\prime}$-free. Conversely, suppose (1) and (2) are true. Let $S$ be a $\gamma^{\prime}$ set of $G-e$. By (2) $S$ does not contain any edge of $N[e]$. Hence $S$ cannot be an edge dominating set of $G$. But, for any edge $f \in N[e], S \cup\{f\}$ is an edge dominating set of $G$. Since $S$ is a minimum edge dominating set for $G-e$, $S \cup\{f\}$ is also a minimum edge dominating set for $G$ and hence $\gamma^{\prime}(G)=|S \cup\{f\}|=\gamma^{\prime}(G-e)+1$. Thus $e$ is $\gamma^{\prime}$-critical.

\subsection{Theorem}

Let $G$ be a graph and $e \in E(G)$. Then

1) $e$ is $\gamma^{\prime}$-fixed if and only if there exists no edge dominating set of $G-e$ with $\gamma^{\prime}(G)$ edges which is also an edge dominating set of $G$.

2) $e$ is $\gamma^{\prime}$-totally free if and only if every $\gamma^{\prime}$-set of $G$ is a $\gamma^{\prime}$-set of $G-e$.

\section{Proof}

1) Assume that $e$ is $\gamma^{\prime}$-fixed. Suppose there exists an edge dominating set $S$ of $G-e$ with $|S|=\gamma^{\prime}(G)$ which is also an edge dominating set of $G$. Then $S$ is a $\gamma^{\prime}$-set not containing $e$ which is impossible as $e$ is $\gamma^{\prime}$-fixed. The converse is obvious.

2) Let $e$ be $\gamma^{\prime}$-totally free. Then $e$ does not belong to any $\gamma^{\prime}$-set of $G$ and so every $\gamma^{\prime}$-set $D$ of $G$ is an edge dominating set of $G-e$. Thus $\gamma^{\prime}(G-e) \leq \gamma^{\prime}(G)$. If $\gamma^{\prime}(G-e) \leq \gamma^{\prime}(G)$, then by theorem $2.3, e$ is $\gamma^{\prime}$-free and so $\gamma^{\prime}(G-e)=\gamma^{\prime}(G), D$ is a $\gamma^{\prime}$-set of $G-e$. The converse is obvious.

\subsection{Theorem}

Let $G$ be a connected graph. If a cut edge $e$ of $G$ is $\gamma^{\prime}$ fixed, then $e$ is $\gamma^{\prime+}$-critical

\section{Proof}

Let $S$ be a $\gamma^{\prime}$-set of G. Let $e$ be a cut edge that is $\gamma^{\prime}$ fixed. Then $e$ belongs to every $\gamma^{\prime}$-set. Since $e$ is a cut edge, $G-e$ is a disconnected graph with at least two components $G^{\prime}$ and $G^{\prime \prime}$. Let $e^{\prime}$ and $e^{\prime \prime}$ be the neighbors of $e$ in $G^{\prime}$ and $G^{\prime \prime}$ respectively. Therefore $D=(S-e) \cup\left\{e^{\prime}, e^{\prime \prime}\right\}$ is a minimum edge dominating set of $G-e$ so that $\gamma^{\prime}(G-e)=\gamma^{\prime}(G)+1$. Hence $e$ is $\gamma^{\prime+}$-critical.

\subsection{Theorem}

An edge $e$ in a graph $G$ is $\gamma^{\prime+}$-critical if and only if

1) $e$ is not isolated edge

2) $e$ is $\gamma^{\prime}$-fixed and

3) There is no edge dominating set for $G-N[e]$ having $\gamma^{\prime}(G)$ edges which also dominates $N[e]$.

\section{Proof}

If $e$ is $\gamma^{\prime+}$-critical, then $\gamma^{\prime}(G-e)=\gamma^{\prime}(G)+1$, by lemma 2.1. Clearly $e$ is not an isolated edge. If $S$ is a $\gamma^{\prime}$-set of $G-N[e]$ having $\gamma^{\prime}(G)$ edges which also dominates $N(e)$ then $\gamma^{\prime}(G-e) \leq \gamma^{\prime}(G)$, a contradiction. Thus no edge dominating set of $G-N[e]$ having $\gamma^{\prime}(G)$ edges can dominate $N(e)$. By Theorem 2.4, $e$ is $\gamma^{\prime}$-fixed. The converse is obvious.

We now investigate relationships between, $\gamma^{\prime}$-free edges, $\gamma^{\prime}$-totally free edges and graphs which are class 1 and class 2 . 


\subsection{Theorem}

If $G$ is a graph without isolated edges, then $G$ is of class 2 if and only if $G$ has $\gamma^{\prime}$-totally free edges.

\section{Proof}

Suppose $G$ has a $\gamma^{\prime}$-totally free edge $e$. By Theorem 2.4 (2), $G$ is of class 2. Conversely, suppose $G$ is of class 2 . Then there exists an edge $e$ which is not in any $\gamma^{\prime}$-set. Hence every $\gamma^{\prime}$-set of $G$ is also a $\gamma^{\prime}$-set of $G-e$ so that $e$ is $\gamma^{\prime}$-totally free.

\subsection{Theorem}

\section{Proof}

Let $G$ be a connected graph. If $G$ has a $\gamma^{\prime}$-fixed edge, then it has a $\gamma^{\prime}$-totally free edge.

Suppose $G$ has a $\gamma^{\prime}$-fixed edge $e$. Then $e$ belongs to every $\gamma^{\prime}$-set.

Claim: No neighbor of $e$ belongs to any $\gamma^{\prime}$-set of $G$. Suppose at least one of its neighbor say $e^{\prime}$ belongs to a $\gamma^{\prime}$ set $D$. Let $e=u v$ and $e^{\prime}$ be incident with $u$. Then $D_{1}=D-\{e\} \cup\left\{e^{\prime \prime}\right\}$, where $e^{\prime \prime}$ is any edge incident with $v$ is an edge dominating set of $G-e$ with $\gamma^{\prime}$-edges which is also an edge dominating set of $G$. But by Theorem 2.6, this is a contradiction, since $e$ is a $\gamma^{\prime}$-fixed edge. Therefore no neighbor of $e$ belongs to any $\gamma^{\prime}$-set of $G$. Thus neighbors of $e$ are all $\gamma^{\prime}$-totally free in $G$.

We now investigate the class of graphs which are $d s^{\prime^{+}}$, $d s^{\prime}$-critical.

\subsection{Lemma}

Let $e \in E(G)$. If $e$ is $\gamma^{\prime}$-totally free and $G-e$ is of class 1 , then $d s^{\prime}(G)=d s^{\prime}(G-e)+1$.

\section{Proof}

Since $e$ is $\gamma^{\prime}$-totally free, by Theorem 2.4,

$$
\gamma^{\prime}(G)=\gamma^{\prime}(G-e)
$$

Since $e$ is $\gamma^{\prime}$-totally free, by Theorem 2.3, $G$ is of class 2 and so

$$
d s^{\prime}(G)=\gamma^{\prime}(G)+1
$$

Since $G-e$ is of class 1 , we have

$$
d s^{\prime}(G-e)=\gamma^{\prime}(G-e)
$$

From Equations (1), (2) and (3), we have

$$
d s^{\prime}(G)=d s^{\prime}(G-e)+1 \text {. }
$$

\subsection{Lemma}

Let $e \in E(G)$. If $e$ is $\gamma^{\prime}$-totally free and $G-e$ is of class 2 , then $d s^{\prime}(G)=d s^{\prime}(G-e)$.

\section{Proof}

If $e$ is $\gamma^{\prime}$-totally free, then by Theorem 2.4,

$$
\gamma^{\prime}(G)=\gamma^{\prime}(G-e)
$$

Since $G$ and $G-e$ are of class 2, we have

$$
d s^{\prime}(G)=\gamma^{\prime}(G)+1
$$

and

$$
d s^{\prime}(G-e)=\gamma^{\prime}(G-e)+1
$$

From equations (1), (2) and (3), we have

$$
d s^{\prime}(G)=d s^{\prime}(G-e) \text {. }
$$

\subsection{Lemma}

Let $e$ be an edge of $G$. If $e$ is $\gamma^{\prime}$-free and $G-e$ is of class 1 , then $d s^{\prime}(G)=d s^{\prime}(G-e)$ or $d s^{\prime}(G)=d s^{\prime}(G-e)+1$.

\section{Proof}

Suppose $e$ is a $\gamma^{\prime}$-free edge. In any case $G$ is either of class 1 or class 2 .

Case (1). $G$ is of class 1 .

Let $S$ be a $\gamma^{\prime}$-set of $G-e$. If $S$ does not contain any neighbor of $e$, then every neighbor of $e$ is $\gamma^{\prime}$-totally free in $G-e$. This implies that $G-e$ is of class 2 . But this is a contradiction and so $S$ must contain a neighbor of $e$. Then by theorem 2.4, $\gamma^{\prime}(G)=\gamma^{\prime}(G-e)$. Since $G$ and $G-e$ are of class 1 , we have

$$
d s^{\prime}(G)=\gamma^{\prime}(G)=\gamma^{\prime}(G-e)=d s^{\prime}(G-e) .
$$

Case (2). $G$ is of class 2 .

Since $G-e$ is of class 1 , then by a similar argument, $S$ must contain a neighbor of $e$. Since $G$ is of class 2, we have $d s^{\prime}(G)=\gamma^{\prime}(G)+1=\gamma^{\prime}(G-e)+1=d s^{\prime}(G-e)+1$.

\subsection{Lemma}

Let $e$ be an edge of $G$. If $e$ is $\gamma^{\prime}$-free and $G-e$ is of class 2 , then $d s^{\prime}(G)=d s^{\prime}(G-e), d s^{\prime}(G)=d s^{\prime}(G-e)+1$ or $d s^{\prime}(G)=d s^{\prime}(G-e)-1$.

\section{Proof}

Case (1). $G$ is of class 1 .

Let $S$ be a $\gamma^{\prime}$-set of $G-e$. We have the following cases:

Subcase (1). $S$ contains a neighbor of $e$.

Now $\gamma^{\prime}(G)=\gamma^{\prime}(G-e)$. Since $G$ is of class 1 and $G-e$ is of class 2 , we have $d s^{\prime}(G-e)=d s^{\prime}(G)+1$.

Subcase (2). $S$ does not contain a neighbor of $e$.

Now $\gamma^{\prime}(G)=\gamma^{\prime}(G-e)+1$. Since $G-e$ is of class 2 and $G$ is of class 1 , we have $d s^{\prime}(G)=d s^{\prime}(G-e)$.

Case (2). $G$ is of class 2 .

By an argument similar to that in case (1), we have $d s^{\prime}(G)=d s^{\prime}(G-e)$ or $d s^{\prime}(G)=d s^{\prime}(G-e)+1$.

\subsection{Lemma}

Let $e$ be an edge of $G$. If $e$ is $\gamma^{\prime}$-fixed and $G-e$ is of class 1 , then $d s^{\prime}(G)=d s^{\prime}(G-e)$.

\section{Proof}

If $e$ is $\gamma^{\prime}$-fixed, then by Theorem 2.8, all of its neighbors are $\gamma^{\prime}$-totally free. Then by Theorem $2.7, G$ is of 
class 2 and hence

$$
d s^{\prime}(G)=\gamma^{\prime}(G)+1
$$

As $e$ is $\gamma^{\prime}$-fixed, by Theorem 2.4, $\gamma^{\prime}(G) \neq \gamma^{\prime}(G-e)$. If $\gamma^{\prime}(G)>\gamma^{\prime}(G-e)$, then $e$ is $\gamma^{\prime}$-critical. Then by Lemma $2.11, e$ is $\gamma^{\prime}$-free and this is a contradiction. Therefore $\gamma^{\prime}(G)=\gamma^{\prime}(G-e)-1$. Since $G$ is of class 2 and $G-e$ is of class 1 , we have $d s^{\prime}(G-e)=d s^{\prime}(G)$.

\subsection{Lemma}

Let $e \in E(G)$. If $e$ is $\gamma^{\prime}$-fixed and $G-e$ is of class 2, then $d s^{\prime}(G)=d s^{\prime}(G-e)-1$.

\section{Proof}

By an argument analogous to that in Lemma 2.13, since $G-e$ is of class 2, we have $d s^{\prime}(G)=d s^{\prime}(G-e)-1$.

\subsection{Theorem}

Let $G$ be a graph without isolated edges. An edge $e$ in $G$ is $d s^{\prime}$-critical if and only if one of the following holds.

1) $e$ is $\gamma^{\prime}$-totally free and $G-e$ is of class 1 .

2) $e$ is $\gamma^{\prime}$-free, $G$ is of class 2 and $G-e$ is of class 1 .

3) $e$ is $\gamma^{\prime}$-free and both $G$ and $G-e$ are of class 2 .

\section{Proof}

Suppose $e$ is $d s^{\prime}$-critical. Then

$$
d s^{\prime}(G)=d s^{\prime}(G-e)+1
$$

Let $S$ be a $\gamma^{\prime}$-set of $G$. Then we have the following cases:

Case (1). $G$ and $G-e$ are of class 1 .

By (1), $\gamma^{\prime}(G)=\gamma^{\prime}(G-e)+1$. By theorem 2.3, $e$ is $\gamma^{\prime}-$ free and no $\gamma^{\prime}$-set of $G$-e contains any edge of $N(e)$. Now every neighbor of $e$ is $\gamma^{\prime}$-totally free in $G-e$. Therefore $G-e$ is of class 2 , which is a contradiction.

Case (2). $G$ is of class 1 and $G-e$ is of class 2 .

Then Equation (1) becomes $\gamma^{\prime}(G)=\gamma^{\prime}(G-e)+2$. But this is not possible.

Case (3). $G$ is of class 2 and $G-e$ is of class 1 .

Then Equation (1) becomes $\gamma^{\prime}(G)=\gamma^{\prime}(G-e)$. Then either $e$ is $\gamma^{\prime}$-free or $\gamma^{\prime}$-totally free.

Case (4). $G$ and $G-e$ are of class 2 .

In this case, Equation (1) becomes $\gamma^{\prime}(G)=\gamma^{\prime}(G-e)+1$. Then by theorem 2.3, $e$ is $\gamma^{\prime}$-free.

From Lemmas 2.9, 2.11 and 2.12, the converse is true.

\subsection{Theorem}

Let $G$ be a graph without isolated edges. An edge $e$ in $G$ is $d s^{\prime^{+}}$-critical if and only if one of the following holds.

1) $e$ is $\gamma^{\prime}$-free, $G$ is of class 1 and $G-e$ is of class 2 .

2) $e$ is $\gamma^{\prime}$-fixed and $G-e$ is of class 2 .

\section{Proof}

Suppose $e$ in $G$ is $d s^{\prime^{+}}$-critical. Hence

$$
d s^{\prime}(G)=d s^{\prime}(G-e)-1
$$

Let $S$ be a $\gamma^{\prime}$-set of $G$. Then we have the following cases:
Case (1). $G$ and $G$-e are of class 1 .

From equation (1) $\gamma^{\prime}(G)=\gamma^{\prime}(G-e)-1$ and so $G$ is $\gamma^{\prime+}$-critical. Hence by Theorem 2.6, $e$ is $\gamma^{\prime}$-fixed, which is a contradiction.

Case (2). $G$ is of class 1 and $G-e$ is of class 2 .

Now equation (1) becomes $\gamma^{\prime}(G)=\gamma^{\prime}(G-e)$. Then $S$ must contain a neighbor of $e$. Since $G$ is of class $1, e$ is $\gamma^{\prime}$-free.

Case (3). $G$ is of class 2 and $G-e$ is of class 1 .

Then Equation (1) becomes $\gamma^{\prime}(G)=\gamma^{\prime}(G-e)-2$, which is not possible.

Case (4). $G$ is of class 2 and $G-e$ is of class 2 .

In this case, Equation (1) becomes $\gamma^{\prime}(G)=\gamma^{\prime}(G-e)-1$. Then by Theorem 2.4, $e$ is $\gamma^{\prime}$-fixed.

Conversely, suppose if (1) or (2) is true. Then by case (1) of Lemma 2.12 and Lemma 2.14, the result follows.

\section{Edge-Domsaturation Number of a Graph}

\section{Theorem}

For any connected unicyclic graph $G=(V, E)$ with cycle $C, d s^{\prime}(G)=q-\Delta^{\prime}(G)+1$ if and only if one of the following holds.

1) $C=C_{3}=\left(u_{1}, u_{2}, u_{3}, u_{1}\right), \operatorname{deg} u_{1} \geq 3, \operatorname{deg} u_{2} \geq 3$, $\operatorname{deg} u_{3}=2, \operatorname{deg} u_{u \in N\left[u_{1}\right] \cap(V-C)} \leq 2$ and there exists $w \in V-C$ such that $d\left(u_{i}, w\right) \leq 2, i=1,2$.

2) $C=C_{3}=\left(u_{1}, u_{2}, u_{3}, u_{1}\right), \operatorname{deg} u_{1} \geq 4, \operatorname{deg} u_{2}=2$, $\operatorname{deg} u_{3}=2$, exactly one vertex $w$ not on $C$ has $\operatorname{deg} w \geq 2$ and remaining vertices are pendent vertices.

\section{Proof}

Suppose $d s^{\prime}(G)=q-\Delta^{\prime}(G)+1$.

Let $C=C_{k}=\left(u_{1}, u_{2}, \cdots, u_{k}, u_{1}\right)$ be the unique cycle in $G$.

If $C=C_{k}$, then $d s^{\prime}(G)=\lceil q / 3\rceil<q-1$ for all $n \geq 3$ and so $G \neq C_{k}$.

Let $S$ denote the set of all pendent edges of $G$ and let $|S|=t$.

Claim 1: $t \leq \Delta^{\prime}(G)-2$. Since $E-(S \cup\{e\})$ is an edge dominating set for any edge $e$ of $C, \gamma^{\prime}(G) \leq q-t-1$. For any pendent edge $f, E-(S \cup\{g, e\}) \cup\{f\}$ is an edge dominating set of $\mathrm{G}$ containing $f$. Here $g$ is an edge adjacent to $f$ and $e$ is any edge of the cycle. Hence $d s^{\prime}(G) \leq q-t-1$, so that $t \leq \Delta^{\prime}(G)-2$.

Claim 2: $e=u v$ is an edge with degree $\Delta^{\prime}$. Then either $u$ or $v$ lies on $C_{k}$.

Now let $G \neq C_{k}$ and $e=u v$ be an edge of maximum degree $\Delta^{\prime}$. If $e \in \mathrm{C}_{\mathrm{k}}$, then for some edge $e^{\prime} \in C_{k}, G-e^{\prime}$ is a tree $T$ of $G$ with at least $\left(\Delta^{\prime}(G)+1\right)$ pendent edges. If $X$ is the set of all pendent edges of $G-e$, then $|X| \geq \Delta^{\prime}(G)+1$. Then $E(T)-X$ is an edge dominating set of cardinality at most $q-\Delta^{\prime}(G)-1$. Therefore $d s^{\prime}(G)<q-\Delta^{\prime}(G)+1$, which is a contradiction.

Case (1). $u$ or $v$ lies on $C$. 
Claim 3: $G-C_{\boldsymbol{k}}$ is the union of $P_{1}$ and $P_{2}$. Suppose not. Then, $G-C_{\boldsymbol{k}}$ contains $P_{\boldsymbol{k}}=x_{1} x_{2} \cdots x_{K}, k \geq 3$. Suppose $u=u_{1}$ lies on $C_{K}$. Let $T_{u_{1}}$ be the maximal tree rooted at $u_{1}$ not containing any edge of $C_{k}$. Clearly $T_{u_{1}}$ has at least $\Delta^{\prime}(G)-2$ pendent edges, say $S$. Then

$E(G)-\left(S \cup\left\{u_{1} u_{2}, u_{k} u_{1}, u_{i} x_{1}\right\}\right), i=1,2,3, \cdots, k$ is an edge dominating set of cardinality less than $q-\Delta^{\prime}(G)$. Therefore $d s^{\prime}(G)<q-\Delta^{\prime}(G)+1$, which is a contradiction.

In this case, $G$ has at least $\Delta^{\prime}-2$ pendent edges. Let $W$ be the set of these pendent edges. Further $d s^{\prime}\left(C_{k}\right)=\lceil k / 3\rceil$ and let $Y$ denote a $\gamma^{\prime}$-set of $C_{k}$. Let $Z=E\left(C_{k}\right)-Y$. If $k>$ 4, then $E(G)-W-Z$ is an edge dominating set of cardinality less than $q-\Delta^{\prime}(G)$. Hence $C_{k}=C_{3}$ or $C_{4}$. Since $t \geq \Delta^{\prime}(G)-2$. By claim $1, t=\Delta^{\prime}(G)-2$.

Subcase (1). $C=C_{3}=\left(u_{1}, u_{2}, u_{3}, u_{1}\right)$

$G-C_{3}$ is the union of $P_{1}$ and $P_{2}$. Also $u$ or $v$ lies on $C$. Let $u=u_{1}$. Therefore $G-C_{3}$ contains at least one $P_{2}$. Since $t=\Delta^{\prime}(G)-2$, no other vertex other than $u$ and $v$ has degree $>3$.

If $G-C_{3}$ is the union of $P_{2}^{\prime} s$ alone, then $\left\{x_{1} x_{2}, u_{i} u_{j}\right\}$ or $\left\{u_{1} x_{1}, u_{i} u_{j}\right\}, i \neq j i, j=1,2,3$ is an edge dominating set and every edge lies in a $\gamma^{\prime}$-set. Therefore $d s^{\prime}(G)=q-\Delta^{\prime}(G)$.

If $G-C_{3}$ is the union of $P_{1}^{\prime} s$ and $P_{2}^{\prime} s$, then from Theorem 1.5, $\gamma^{\prime}(G)=q-\Delta^{\prime}(G)$. But pendent edges adjacent to $u_{1}$ does not lie in any $\gamma^{\prime}$-set. Therefore $d s^{\prime}(G)=q-\Delta^{\prime}(G)+1$.

Subcase (2). $C=C_{4}=\left(u_{1}, u_{2}, u_{3}, u_{4}, u_{1}\right)$

As in subcase (1), $G-C_{4}$ also contains $P_{2}$. Then

$E(G)-W-\left\{u_{1} u_{2}, u_{1} u_{4}, u_{2} u_{3}\right\}$ is an edge dominating set of cardinality $<q-\Delta^{\prime}(G)$. Therefore

$d s^{\prime}(G)<q-\Delta^{\prime}(G)+1$.

Case (2). $u$ and $v$ lies on C.

Claim 4: $G-C_{k}$ is the union of $P_{1}$ and $P_{2}$.

Suppose not. Then, $G-C_{k}$ contains $P_{k}=x_{1} x_{2} \cdots x_{k}$, $k \geq 3$. Suppose $e=u_{1} u_{2}$ lies on $C_{k}$. Let $T_{u_{1} u_{2}}=T_{u_{1}} \cup T_{u_{2}} \cup\left\{u_{1}, u_{2}\right\}$.

Clearly $T_{u_{1} u_{2}}$ has at least $\Delta^{\prime}(G)-2$ pendent edges, say $P$.

Then $E(G)-P-\left\{u_{i} x_{1}, u_{k} u_{1}, u_{2} u_{3}\right\}, i=1,2 \cdots k$ is an edge dominating set of cardinality less than $q-\Delta^{\prime}(G)$. Therefore $d s^{\prime}(G)<q-\Delta^{\prime}(G)+1$, which is a contradiction.

As in case (1), $t=\Delta^{\prime}(G)-2$. Let $e=u_{1} u_{2}$ be an edge of maximum degree.

Subcase (1). $C=C_{3}=\left(u_{1}, u_{2}, u_{3}, u_{1}\right)$

In this case, from Theorem 1.5, (3), $u_{3} u_{1}$ does not belong to any $\gamma^{\prime}$-set. Therefore $d s^{\prime}(G)=q-\Delta^{\prime}(G)+1$.

Subcase (2). $C=C_{4}=\left(u_{1}, u_{2}, u_{3}, u_{4}, u_{1}\right)$

From Theorem 1.5, there does not exist an edge dominating set of cardinality $q-\Delta^{\prime}(G)$.
The converse is obvious.

\section{Well-Edge Dominated Graph}

A graph $G$ is called well dominated if all minimal dominating sets have the same cardinality. This concept was introduced by Finbow, Hartnell and Nowakowski [6].

\subsection{Definition}

A graph $G$ is well-edge dominated if every minimal edge dominating set of $G$ has the same cardinality.

\subsection{Lemma}

If $G$ is a well-edge dominated graph and $e$ is an edge of $G$, then there exists a minimum edge dominating set containing $e$ and a minimum edge dominating set not containing $e$.

Proof

To obtain an edge dominating set containing $e$, place $e$ in the set $D$, delete $N[e]$ from $G$ and continue in this greedy fashion until there are no edges left. Then $D$ is minimal and since $G$ is well-edge dominated, it is minimum.

To obtain a minimum edge dominating set not containing $e$, we use the same greedy method except that we use a neighbor of $e$ as our initial edge in $D$.

\subsection{Theorem}

If $G$ is well-edge dominated, then $G$ is of class 1 .

\section{Proof}

From the above lemma, it is clear that every edge belongs to any one of the $\gamma^{\prime}$-set. Therefore $G$ is of class 1 .

\section{REFERENCES}

[1] F. Harary, "Graph Theory," Addison-Wesley Publishing Company, Boston, 1969.

[2] T. W. Haynes, S. T. Hedetniemi and P. J. Slater, "Fundamentals of Domination in Graphs," Marcel Dekker, New York, 1998.

[3] B. D. Acharya, "The Strong Domination Number of a Graph and Related Concepts," Journal of Mathematical Physics, Vol. 14, No. 5, 1980, pp. 471-475.

[4] S. Arumugam and R. Kala, "Domsaturation Number of a Graph," Indian Journal of Pure and Applied Mathematics, Vol. 33, No. 11, 2002, pp. 1671-1676.

[5] S. Arumugam and S. Velammal, "Edge Domination in Graphs," Taiwanese Journal of Mathematics, Vol. 2, No. 2, 1998, pp. 173-179.

[6] A. Finbow, B. L. Hartnell and R. Nowakowski, "Well Dominated Graphs: A Collection of Covered Ones," Ars Combinatoria, Vol. 25, No. A, 1988, pp. 5-10. 